\title{
A Multi-centered Study to Evaluate the Efficacy and Safety of Amitriptyline and Mecobalamine in the Management of Neuropathic Pain
}

\author{
Dr. Mayuresh Dilip Kiran ${ }^{1 *}$, Shaheen Naseem Sheikh ${ }^{2}$, Lalit Jeeevan Pawaskar ${ }^{3}$ \\ ${ }^{1}$ Vice-President, Medical Services and Pharmacovigilance, Centaur Pharmaceuticals Pvt. Ltd. \\ ${ }^{2}$ Research Associate, Pharmacovigilance, Centaur Pharmaceuticals Pvt. Ltd. \\ ${ }^{3}$ Executive, Pharmacovigilance, Centaur Pharmaceuticals Pvt. Ltd.
}

\section{Corresponding Author}

\author{
Dr. Mayuresh Dilip Kiran \\ Vice-President, Medical Services and Pharmacovigilance. \\ Centaur Pharmaceuticals Pvt. Ltd., Mumbai, India. \\ Email: mayuresh_kiran@ rediffmail.com
}

\begin{abstract}
Introduction: Neuropathic Pain (NP) is known as 'nerve pain'- is a type of chronic pain that occurs when nerve in the central nervous system becomes damage or injured. An FDC of Amitriptyline a neuroanalgesia and Mecobalamine (Vitamin $\mathrm{B}_{12}$ ) a damage nerve rejuvenator have an important role in management of NP. Methodology: Of 786 registered, 666 patients completed the study. Efficacy assessment was made by reduction in mean VAS score and $\geq 50 \%$ reduction in NP was calculated at conclusion visit and used to determine the corresponding NNT which was calculated by using risk reduction. Safety assessment was made by inspecting the adverse events during trial. Results: Reduction in mean VAS score was from 7.3 (baseline) to 5.6 (day 30) and 3.9 (day 45). Nearly all the patients had experience $\geq 50 \%$ reduction in NP and NNT score of 1.21 which was calculated by using risk reduction parameter at conclusion visit. Overall 236 patients experience adverse events and were of mild to moderate intensity, sedation and drowsiness being dominantly seen. Conclusion: A combination of Amitriptyline and Mecobalamine was efficacious, safe and well-tolerated in management of Neuropathic pain (NP).
\end{abstract}

Keywords: Neuropathic pain Amitriptyline, Mecobalamine, VAS score and NNT score

\section{Introduction}

The International Association for the Study of Pain (IASP) defined Neuropathic Pain (NP) as "pain initiated or caused by a primary lesion or dysfunction of the nervous system." In other words, pain due to nerve damage or somatosensory system disease characterized by motor and sensory disorders. ${ }^{[1],[2]} \mathrm{NP}$ is a chronic pain which has extreme impact on patient's quality of life, which is gradually debilitating. NP can results from numerous causes such as nerve compression, tumors, degenerative diseases, infection, surgery, drugs, among others relating risk factors for pain such as type 2 diabetes. ${ }^{[2],[3]}$

The prevalence of NP conditions is difficult to establish as there are invalidate factors that leads to under-reporting. The prevalence has been reported overall for NP is between $2-11 \%$ on general population, ${ }^{[4],[5]} 26 \%$ patients with type 2 diabetes experience NP, $37 \%$ of patients having chronic low back pain related to neuropathy. ${ }^{[6],[7],[8]}$ Chronic NP is more frequent in females (8\%) than males $(5.7 \%)$ and in $8.9 \%$ patients $>50$ years of age and $5.6 \%$ patients <49 years of age and most commonly affects the lower limbs and lower back, neck and upper limbs. ${ }^{[9],[10]}$

The pharmacological treatment of NP is essential but at the same time the outcome is unpredictable. NP should not be considered as a disease by itself, but considered as a clinical condition which is common to different pathologies. ${ }^{[2],[11],[12]}$ The therapeutic management of NP is based on the clinical scenario and the diagnosis obtained after physical examination. Proper assessment and diagnosis of painful process caused by neuropathy can be key to treatment success. ${ }^{[2]}$ Tricyclic anti-depressants (TCAs) is the class of drugs presents results with extensive evidence in the treatment of NP and are recommended as first- line treatment in the management of neuropathic pain. ${ }^{[13]}$

Amitriptyline is a derivative of dibenzocycloheptadiene and a tricyclic anti-depressant (TCA). The mechanism of action lies on blocking the reuptake of norepinephrine and serotonin to presynaptic level, limiting the hyperalgesia induced by N-methyl-Daspartate agonists, blockade of sodium channels that allows the stabilization of neuronal peripheral level and modulation of 


\section{International Journal of Innovative Research in Medical Science (IJIRMS) Volume 04 Issue 01 Jan 2019, ISSN: 2455-8737, Imp. Factor - 4.102 \\ Available online at $-\underline{w w w . i j i r m s . i n}$}

neuronal hyperactivity at central level, anti-histamine action on the $\mathrm{H} 1$ and $\mathrm{H} 2$ receptors, blockade of alpha receptors that can eliminate pain maintained by noradrenergic stimulation and stimulation of $\mu$-opioid receptors despite the low affinity. ${ }^{[2],[11],[13-}$ 15]

Mecobalamine is a potent and active form of vitamin cobalamine (Vitamin $\mathrm{B}_{12}$ ). It plays a key role in maintaining good health. Its deficiency can lead to anaemia and neuronal dysfunction. Mecobalamine is used in the treatment of diabetic neuropathy, megaloblastic anaemia and degenerative disorder. Mecobalamine works by functioning in the production of a compound called myelin, which covers and protect the nerve fibres. It rejuvenates the damage neuron. Without enough Mecobalamine for 4 weeks results in improvement of peripheral neuropathy and it produce very significant effect after 12 weeks of treatment. An improvement in vibration sense, lower motor neuron weakness and sensitivity to pain is also observed. ${ }^{[16]}$

Amitriptyline, as neuroanalgesia is of a particular value in the management in the management of neuropathic pain. Mecobalamine is the physiologically most active from of Vitamin $\mathrm{B}_{12}$. Mecobalamine causes nerve rejuvenation by promoting myelination, axonal regeneration and restoring diminished neurotransmitter levels. Thus the combination of Amitriptyline and Mecobalamine can be valuable assets in the management of neuropathic pain. However, till now no data is available on the efficacy and safety of the combination of Amitriptyline and Mecobalamine. However this is the first Indian clinical study (AMNEST) which evaluate the efficacy and safety of Amitriptyline and Mecobalamine combination in management of neuropathic pain.

\section{Methodology}

A multi-centered Phase IV clinical trial was conducted at 250 centres in various cities of India. Total 786 patients recruited out of which 666 completed the trial and 120 were lost to follow-up.

\section{Inclusion Criteria}

The study include patients of both the genders of age of and above 18 years. Patients with confirmed diagnosis of NP due to any reason of the following causes are Diabetic Neuropathy, Neuritis, Spondylitis, Chronic Low Back Ache (CLBA), Radiculopathy, and Trigeminal Neuralgia were included in the study. If the patient is diabetic, specific treatment for diabetes with anti-diabetics is being taken. In patients whose NP is not controlled with any other drug or therapy. Only patients who strictly adhere to the protocol were recruited for this Phase IV study.

\section{Exclusion Criteria}

Patients having hypersensitivity to the study drug or excipient of study formulation were excluded from the study. Patients with history of urinary retention (benign prostatic hyperplasia) and patients taking MAO inhibitors were excluded from the study. Patients with hepatic/renal impairment or mentally ill were excluded from the study. Pregnant women and breastfeeding mothers were also excluded from the study.

\section{Study intervention}

Study drug- A film coated bilayered tablet containing a combination of Amitriptyline $10 \mathrm{mg}$ (IR) + Mecobalamine 1500 mcg (SR) was provided by the sponsor free of cost to the patients recruited for the study. Study dosage and administration - Patients were instructed to take one tablet at night for a study period of 45 days.

\section{Study Procedure}

The study stretch was decided to be of 45 days to determine the safety and efficacy of the Amitriptyline and Mecobalamine combination in the management of NP. Patients of NP satisfying the inclusion/exclusion criteria were mustered for the study. Physical examination which include respiratory rate, blood pressure and plus rate was conducted and medical history was taken by the investigators. Patients were dispensed with 5 blister packs containing 10 tablets each blister and were instructed to take in a dose of one tablet at night.

Patients were also evaluated for efficacy by Visual Analogue Scale (VAS) score and it was noted on V1 (Baseline visit) on day 1, V2 (Re-evaluation visit) on day 30 and V3 (Conclusion visit) on day 45. The change in VAS score on visit 2 and visit 3 compared to baseline was evaluate statistically. Fasting and Post- prandial blood glucose was measured at every visit. Patients were also asked to keep daily note for any adverse events that had occurred during study duration of 45 days.

Patients were evaluated by standardized measures of efficacy and are needed to compare efficacy across the study duration. The number needed to treat (NNT) is considered a statistically robust and readily interpretable measure to rank the efficacy of the treatment including analgesics. The NNT is use to compare the efficacy of chronic pain treatment and it is defined as the number of patients that need to be treated for one to receive benefit. Smaller the NNT, fewer the number of patients needed to treat to obtain a beneficial effect. Patients were asked to discontinue the study drug in case of any severe adverse event by the investigator.

\section{Concomitant therapy}

No other analgesics was used apart from the study drug. Any nonpharmacological therapy to alleviate pain was allowed during the trial duration. Every use of concomitant medications was noted.

\section{Efficacy Assessment}

The primary assessment was done to evaluate the reduction in the VAS score on an eleven-point scale (0 to 10$)$ where 0 means no symptoms i.e. patients who are completely cured and 10 means maximum tolerated symptoms. The secondary assessment was done to determine $\geq 50 \%$ reduction in NP from baseline were calculated at day 45 and these values were used to determine the corresponding NNT. NNT was calculated using risk reduction

\section{Safety assessment}

Patients were asked for any adverse event at each visit and if present were noted in the case record form (CRF) during each visit. These adverse events were classified into serious and non-serious adverse events. Naranjo's scale of probability was used to classify the adverse event as drug related or nondrug related. Adverse events were followed up and also treated if it is found to be necessary. 


\section{International Journal of Innovative Research in Medical Science (IJIRMS) Volume 04 Issue 01 Jan 2019, ISSN: 2455-8737, Imp. Factor - 4.102 \\ Available online at $-\underline{w w w . i j i r m s . i n}$}

\section{Regulatory matters}

The study drug in combination has been approved for manufacturing and marketing in 2005. The combination is available under various brands but is classified as schedule $\mathrm{H}$ drug in India, i.e. to be sold in presence of prescription of registered medical practitioners only. All the patients participating in this study read and signed the informed consent form, voluntarily. The protocol, case record form, informed consent form, investigators undertaking, investigators $\mathrm{CV}$, investigators medical registration certificates (including post-graduation certificates) and ethics committee registration certificates were submitted to the office of Drug Controller General of India (DCGI), Central Drugs Standard Control Organization (CDSCO).

\section{Results}

A total of 786 patients were recruited from 250 centres all across India, 666 patients completed the study and were analysed.

\section{Efficacy Analysis}

A total number of 666 patient's data was received. The primary outcome of the study was change in mean VAS score from V1 (Baseline visit) on day 1 to V3 (Conclusion visit) on day 45. The change in mean VAS score was measured and was found to be 7.39 at $\mathrm{V} 1$ which reduced to 5.60 at V2 and further reduced to 3.99 at V3 (Figure 1).



Figure 1: Change in Mean VAS Score from the baseline

The secondary outcome of the study was patients achieving $\geq 50 \%$ reduction in NP calculated at V3 (Conclusion Visit) on day 45 and these value was used to determine NNT. At V1 (Baseline visit) on day 1, out of 786 patients $666(84.73 \%)$ patients had a pain score of $>5 \%$. During V2 (Re-evaluation visit) and V3 (Conclusion Visit), $\geq 50 \%$ reduction in pain was demonstrated in $63.99 \%$ and $82.44 \%$ of patients, respectively (Figure 2). These results suggested that majority of the patients have experienced $\geq 50 \%$ reduction in pain, hence the study drug showed an NNT score of 1.21 calculated by using risk reduction of 0.82 .

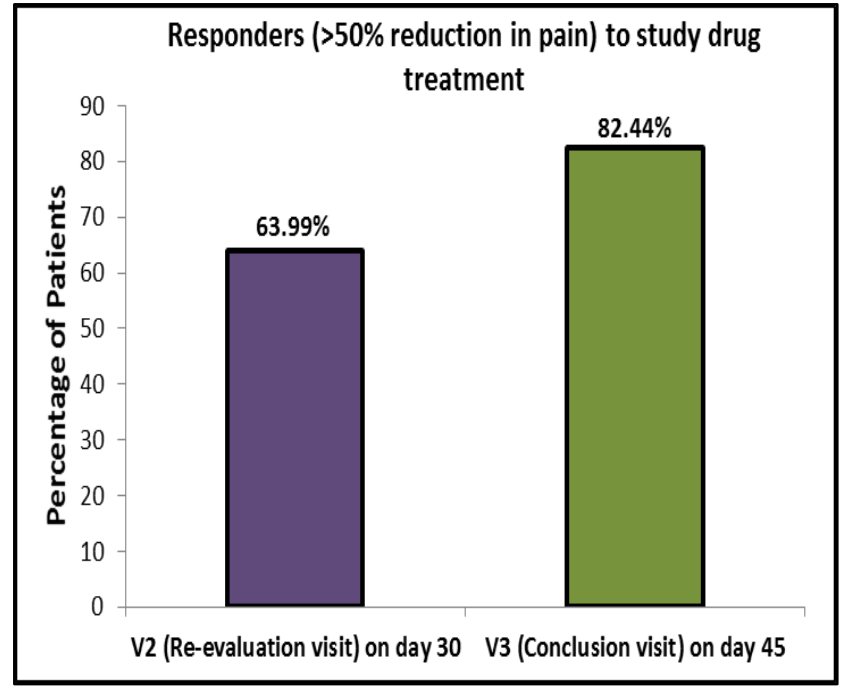

Figure 2: Responders ( $>50 \%$ reduction in pain) to study drug treatment

\section{Safety Analysis:}

No serious adverse events was observed during the study duration. The overall incidence of reported study drug related adverse effects was seen in 236 patients. The list of adverse events with the number of episodes is mentioned in Table 1.

Table no. 1: Adverse events, no. of episodes, no. of patients and percentage of patients experience from total population.

\begin{tabular}{|l|c|c|}
\hline Adverse event & $\begin{array}{c}\text { No. of } \\
\text { patient }\end{array}$ & $\begin{array}{c}\text { Percentage of } \\
\text { patients }\end{array}$ \\
\hline Sedation and Drowsiness & 166 & $24.92 \%$ \\
\hline Dizziness & 33 & $5 \%$ \\
\hline Dry mouth & 57 & $8.55 \%$ \\
\hline GI irritation & 48 & $7.2 \%$ \\
\hline Constipation & 42 & $6.30 \%$ \\
\hline Total & 236 & $35.42 \%$ \\
\hline
\end{tabular}

\section{Discussion}

Several combination in neuroanalgesics with neuron rejuvenators are available in the market and quite popular. This is the first study evaluating safety and efficacy of Amitriptyline and Mecobalamine combination in management of NP in Indian patients. The strength of the study is both the parameters which leads to reduction in NP i.e. change in mean VAS score and NNT were clinically studied over the study duration of 45 days.

At V1 (Baseline visit) on day 1 the mean VAS score was found out to be 7.39 which reduced to 5.6 at V2 (Re-evaluation visit), and further reduced to 3.99 at V3 (Conclusion Visit). Patients were also demonstrated on NNT for Amitriptyline and Mecobalamine combination in management of NP. The outcome of the study was achieving $>50 \%$ reduction in NP. At V1 (Baseline visit) on day 1 , out of 786 patients $666(84.73 \%)$ patients had a pain score of $>5 \%$. During V2 (Re-evaluation visit) and V3 (Conclusion Visit), $\geq 50 \%$ reduction in pain was demonstrated in $63.99 \%$ and $82.44 \%$ of patients, respectively and hence the study drug showed an NNT score of 1.21 calculated by using risk reduction of 0.82 . Overall 236 patients was observed with study drug related adverse events and were reported. Sedation and Drowsiness, Dizziness, Dry 


\section{International Journal of Innovative Research in Medical Science (IJIRMS) Volume 04 Issue 01 Jan 2019, ISSN: 2455-8737, Imp. Factor - 4.102 \\ Available online at - www.ijirms.in}

mouth, GI irritation and Constipation was the documented adverse event in this study affecting $35.42 \%$ of the study population.

Dongre Yasmin U., et $\mathrm{al}^{[17]}$ conducted an observational, multicenteric, prospective, open-labelled, single-arm clinical study of Sustained-release Pregabaline with Methylcobalamin in neuropathic pain for the duration of 14 days in Indian Patients. The main objective of the study was efficacy and safety data for the combination. Visual analogue scale (0-10) was used to determine the efficacy by measuring the reduction in intensity of pain. Other positive as well as negative symptoms associated with neuropathy was noted. Adverse events were noted to determine the safety of the drugs throughout the study duration. In 14 days there was reduction of $72.3 \%$ in VAS score as compared to the baseline. Both positive and negative symptoms of Peripheral Neuropathy were significantly improved in more than $>50 \%$ patients within 2 weeks. In safety measurement it was found that patients were commonly experienced Giddiness (4.7\%), followed by nausea (2.3\%), sedation $(3.6 \%)$, dizziness $(2.9 \%)$ and drowsiness $(2.3 \%)$ were the most commonly observed adverse effects. It was concluded that fixed dose combination of sustained-release Pregabaline and Methylcobalamin is efficacious as well as safe for the treatment for the treatment of neuropathic pain, with significant improvement in both the positive and negative symptoms associated with neuropathy in Indian patients.

Kalita, J., et al. ${ }^{[18]}$ has conducted single centred, investigators initiated randomised controlled open labelled trial to evaluate the safety and efficacy of Pregabaline (PG) and Amitriptyline (AMT) in CLBA patients. The study was conducted in North India's tertiary care teaching unit. The patients were divided in to two groups AMT and PG group. As per the protocol analysis 77 patients in AMT group and 70 patients in PG group were analysed respectively. The patients were examined at 6 weeks and 14 weeks after the randomization. In each follow-up, the pain severity on 010 Visual Analogue Scale (VAS) and functional disability was measured by Oswestry Disability Index (ODI) version 2 were evaluated. The VAS score and ODI improved in both groups compared to baseline at 6 and 14 weeks. In the AMT group, the mean VAS score improved from 6.7 to 4.0 at 6 weeks and to 2.8 at 14 weeks. Similarly in the PG group the mean VAS score improved from 6.7 to 4.5 at 6 weeks and 3.8 at 14 weeks. The VAS score was not significantly different at 6 weeks $(P=0.06)$ between both the group but at 14 weeks the patients in AMT group improved significantly compared to PG group $(P=0.03)$. The ODI was not significantly different between both groups at 6 weeks $(P$ $=0.23)$ and 14 weeks $(P=0.09)$. At 14 weeks, the improvement in VAS score was found to be more than 50\% (57.3\% Vs. 39.2\%; $P=$ $0.01)$ and ODI was found to be more than $20 \%(65.0 \% \mathrm{Vs}$. $49.5 \%$; $P=0.03$ ) was more frequent in the AMT group as compared to the PG group. As per protocol analysis, the reduction in pain $(86 \% \mathrm{Vs}$. $54 \%, \mathrm{P}=0.05)$ and disability $(84 \%$ Vs. $63 \%, \mathrm{P}=0.05)$ was significantly higher in AMT group compared to PG group. The side effects were noted in 18 patients in the AMT group and 21 in the PG group $(P=0.48)$. The patients in AMT group had sedation (10) and dry mouth (3) whereas in the PG group, the common side effects were vertigo (6) and sedation (4). The combined side effects between the two groups were not significantly different. However, it was concluded that AMT and PG group are effective in CLBA but AMT group reduced pain and disability more significantly compared to PG group.
Morello, Candis M., et $\mathrm{al}^{[19]}$ has conducted randomized prospective, double-blinded, crossover study to compare the efficacy of Gabapentin (GABA) with Amitriptyline (AMT) on Diabetic Peripheral Neuropathy Pain (DNP). Twenty-eight patients were referred by a primary care providers Patients with stable glycemic control and NP randomized to 6 weeks of treatment with GABA or AMT with a one week washout period. The main outcome of the study was pain relief and measured by pain scale with verbal description with patients and global pain score assessment at the end of treatment. Patients reached maximum dosage with GABA and AMT was $65 \%$ and $54 \%$ respectively. The mean score analysis showed pain relief with both the drugs and was not significantly different $(P=0.26)$. Global data obtain from 21 patients out of 25 enrolled patients who completed the study. Severe or moderate pain relief was observed in 11 of 21 patients (52\%) with GABA and 14 of 21 patients (67\%) with AMT. Although both the drugs provide pain relief, mean pain score and global pain score data indicates no significant difference between GABA and AMT. GABA may be an alternative in the treatment of DNP, but does not have considerable advantage over AMT and is more expensive. The severity of adverse effects were similar with both the drugs.

\section{Conclusion}

A combination of Amitriptyline and Mecobalamine was efficacious, safe and well-tolerated in management of Neuropathic pain (NP) due to Diabetic Neuropathy, Neuritis, Spondylitis, CLBA, Radiculopathy and Trigeminal Neuralgia.

\section{Acknowledgements and Disclosures}

AMNEST study was conducted as a part of Pharmacovigilance activity for Amnurite Tablet marketed by Centaur Pharmaceuticals Pvt. Ltd. in accordance with Pharmacovigilance Program of India (PvPI).

\section{Reference}

[1] Koltzenburg, Martin, and John Scadding. "Neuropathic pain." Current opinion in neurology 14.5 (2001): 641647.

[2] Amorim, Daniela. "Pharmacological treatment of neuropathic pain: review of oral and topical therapy recommendations." Int J Clin Neurosci Mental Health 2 (2015): 4.

[3] Ballantyne J, Cousins M, Giamberardino M, Jamison R, McGrath P, Rajagopal M, Smith M, Sommer C, Wittink H. Diagnosis and classification of neuropathic pain. Seatle: IASP 2010. Vol18.

[4] Clark, J. David. "Chronic pain prevalence and analgesic prescribing in a general medical population." Journal of pain and symptom management 23.2 (2002): 131-137.

[5] Koleva, Daniela, et al. "Pain in primary care: an Italian survey." The European Journal of Public Health 15.5 (2005): 475-479.

[6] Davies, Mark, et al. "The prevalence, severity, and impact of painful diabetic peripheral neuropathy in type 2 diabetes." Diabetes care 29.7 (2006): 1518-1522. 
[7] Freynhagen, Rainer, et al. "Screening of neuropathic pain components in patients with chronic back pain associated with nerve root compression: a prospective observational pilot study (MIPORT)." Current Medical Research and Opinion 22.3 (2006): 529-537.

[8] Bennett, Michael I., et al. "Prevalence and aetiology of neuropathic pain in cancer patients: a systematic review." Pain 153.2 (2012): 359-365.

[9] Colloca, Luana, et al. "Neuropathic pain." Nature Reviews Disease Primers 3 (2017): 17002.

[10] Bouhassira, Didier, et al. "Prevalence of chronic pain with neuropathic characteristics in the general population." Pain 136.3 (2008): 380-387.

[11] Gálvez Mateos R. Manual práctico del dolor neuropático. Elservier España, S.L. Granada. 2010.

[12] Haanpää M, Hietaharju A. Central neuropathic pain. Guide to pain management in low-resource settings. Seatle: IASP 25 (2010).

[13] Moulin, D. E., et al. "Pharmacological management of chronic neuropathic pain-consensus statement and guidelines from the Canadian Pain Society." Pain Research and Management 12.1 (2007): 13-21.
[14] Attal, N., et al. "EFNS guidelines on the pharmacological treatment of neuropathic pain: 2010 revision." European journal of neurology 17.9 (2010): 1113.

[15] Ryder, Sally-Ann, and Catherine F. Stannard. "Treatment of chronic pain: antidepressant, antiepileptic and antiarrhythmic drugs." Continuing Education in Anaesthesia, Critical Care \& Pain 5.1 (2005): 18-21.

[16] Gupta JK, Sana Q.S. potential benefits of methylcobalamin: A Review. Austin J Pharmacol Ther. 3.3 (2015): 1076.

[17] Dongre, Yasmin U., and Onkar C. Swami. "sustainedrelease pregabalin with methylcobalamin in neuropathic pain: an Indian real-life experience." International journal of general medicine 6 (2013): 413.

[18] Kalita, J., et al. "An open labeled randomized controlled trial of pregabalin versus amitriptyline in chronic low backache." Journal of the neurological sciences 342.1 (2014): 127-132.

[19] Morello, Candis M., et al. "Randomized double-blind study comparing the efficacy of gabapentin with amitriptyline on diabetic peripheral neuropathy pain." Archives of internal medicine 159.16 (1999): 1931-1937. 